\title{
Studien auf fragwürdiger Basis
}

\author{
Urs Metzgera, Stefan Felder ${ }^{\mathrm{b}}$ für das Appraisal Komitee Swiss Medical Board \\ a Professor em., ehem. Chefarzt Chirurgie, Triemlispital \\ b Prof. Dr. rer. pol., Ordinarius für Health Economics, Universität Basel
}

Die Replik des SMB zur Stellungnahme von swiss orthopaedics zum Review des SMB-Fachberichts «Ruptur des VKB: operative oder konservative Behandlung?» [1] hat die Orthopäden zu einer erneuten Antwort veranlasst. Abgesehen vom mittlerweile üblichen Vorwurf der Inkompetenz an Andersdenkende haben die Autoren "zwei neue und relevante Studien zur Kosten-Nutzwert-Analyse gefunden». Im Gegensatz zur entscheidenden prospektiv-randomisierten Frobell-Studie [2] handelt es sich bei diesen zwei Arbeiten ein und desselben Autorenteams um Modellberechnungen auf fragwürdiger Basis, in denen einesteils die direkten Kosten bis 6 Jahre (Studie 1) und andernteils die lebenslangen (!)

\section{Es handelt sich bei diesen zwei Arbeiten ein und desselben Autorenteams um Modell- berechnungen auf fragwürdiger Basis.}

direkten und indirekten Kosten (Studie 2) mit Hilfe eines Wahrscheinlichkeits-Modells kalkuliert wurden. Als Grundlagen dienten die Daten der Frobell-Studie in Schweden (121 Patienten) und eines US-amerikanischen Registers (Multicenter Orthopaedic Outcomes Network, $M O O N)$ von 807 Patienten. In diesem Register wurden aber ausschliesslich Patienten mit primärer Kreuzbandrekonstruktion erfasst. In der ersten Arbeit (Cost-Effectiveness Analysis of Early Reconstruction Versus Rehabilitation and Delayed Reconstruction for Anterior Cruciate Ligament Tears) ging es den Autoren um einen Vergleich der frühen mit der verzögerten Kreuzbandplastik ("Objective: To compare the cost-effectiveness of early versus delayed ACLR»). Mit Recht erwähnen die Autoren, dass in der Frobell-Studie 39\% der Patienten im konservativen Therapiearm innerhalb von 2 Jahren und weitere $10 \%$ innerhalb von 2-6 Jahren eine Operation benötigten. Dies bedeutet aber umgekehrt, dass 51\% der Patienten eine Operation erspart blieb! Ein gesundheitsökonomischer Vergleich der Frühoperation mit der Spätoperation ist deshalb nicht zulässig, weil es sich bei der letzten möglicherweise um eine negative Selektion handelt (Selektionsbias). Entscheidend wäre eine prospektive Erfassung der Kosten gemäss intention to treat. Leider machen Frobell et al. in ihrer randomisierten Studie dazu keine Angaben.
In der zweiten Arbeit (Societal and Economic Impact of Anterior Cruciate Ligament Tears) haben die Autoren neben den direkten auch die lebenslangen indirekten Kosten infolge Produktivitätsverlust durch Erwerbsunfähigkeit und Krankheitstage kalkuliert und einen Vorteil von USD 50417 zugunsten der Frühoperation (USD 38121) errechnet gegenüber dem konservativen Vorgehen (USD 88538). Abgesehen davon, dass es sehr problematisch ist, US-Lohndaten in das Schweizer Gesundheitssystem zu übertragen, ist es aus klinischer Sicht schlicht nicht nachvollziehbar, dass das konservative Vorgehen - das der Hälfte der Patienten eine Operation erspart - mehr als doppelt so teuer sein sollte. Die in der Studie präsentierten ökonometrischen Schätzungen zum Einkommensverlust aufgrund erheblicher Knieschmerzen und Instabilität vermögen denn auch nicht zu überzeugen.

Ganz im Widerspruch zu den zitierten US-amerikanischen (orthopädischen) Schätzungen und Empfehlungen gelangen Biedert und Farron bezugnehmend auf die SUVA-Daten zum Schluss, «dass in den letzten 10 Jahren viel weniger primäre Kreuzbandrekonstruktionen durchgeführt wurden, weil die Indikationen

\section{Dies bedeutet umgekehrt, dass 51\% der Patien- ten eine Operation erspart blieb!}

der Fachspezialisten schon lange viel zurückhaltender gestellt wurden» und weil dies "auf die allgemeingültige Schweizer best practice zurückzuführen» sei. Sie bestätigen damit die Empfehlungen des SMB-Berichts, wenn auch dieser Trend bereits vor Veröffentlichung desselben im Jahre 2009 einsetzte.

Biedert und Farron beklagen schliesslich den mangelnden Einbezug der Fachgesellschaften bei der Erstellung der SMB-Berichte. In diesem Zusammenhang mag es interessieren, dass das SMB seit diesem Jahr die Mitwirkung aller interessierten Stakeholder weiter ausgebaut hat. Diese werden zu einem öffentlichen Hearing eingeladen und ihre Stellungnahmen in die Berichte aufgenommen.

\footnotetext{
Literatur

1 Metzger U, Bohnenblust H. Replik des Swiss Medical Board zur Stellungnahme von swiss orthopaedics. Schweiz Ärztezeitung. 2014;95(29/30):1092.

2 Frobell et al. BMJ. 2013 Jan 24;346:f232. doi: 10.1136/bmj.f232.
} 